INGENIERIA MECANICA

\title{
Enfoque modular para la dinámica de mecanismos planos
}

MECHANICAL ENGINEERING

\section{Dynamics of planar mechanisms by a modular approach}

\author{
Sebastián Durango *§, Cesar Giraldo *, Gabriel Calle **, Leonardo Mesa*** \\ *Grupo Diseño Mecánico y Desarrollo Industrial, Universidad Autónoma de Manizales, Manizales, \\ Colombia. \\ **Grupo Procesos de Manufactura y Diseño de Máquinas, Universidad Tecnológica de Pereira, \\ Pereira, Colombia. \\ ***CINDETEM, Cámara de Comercio de Dosquebradas, Dosquebradas, Colombia. \\ §sebastiandi@autonoma.edu.co, cesarigiraldo@hotmail.com,gcalle@utp.edu.co,ingenieria@ \\ camado.org.co
}

(Recibido: 19 de Octubre del 2012 - Aceptado: 10 de Diciembre del 2013)

\begin{abstract}
Resumen
Este trabajo trata la dinámica directa de mecanismos de un Grado de Libertad (GDL). Se presenta un método de Propósito General (PG) basado en cadenas en el que un mecanismo es desagregado en grupos estructurales de Assur. Los análisis cinemático y de fuerzas de los grupos de Assur se resuelven individualmente para formar una librería de módulos independientes. Esta propiedad es usada para reducir los parámetros inerciales y las fuerzas exteriores en cada grupo (módulo) que forma el mecanismo. La reducción se propaga de módulo a módulo hasta que se alcanza el eslabón conductor, entonces la dinámica directa se resuelve analizando exclusivamente ese eslabón. El método PG presentado tiene una flexibilidad comparable con los métodos PG basados en juntas. El método es usado en la dinámica directa de un mecanismo de seis barras (Watt) con resultados que son comparables con software comercial para dinámica de Sistemas Multicuerpo (MBS).
\end{abstract}

Palabras clave: Análisis modular, dinámica directa, mecanismos.

\begin{abstract}
This work deals with the forward dynamics of mechanisms with one Degree of Freedom (DOF). There is presented a chain-based General Purpose (GP) method in which a mechanism is disaggregated into structural Assur groups. Kinematic and force analyses of Assur groups are individually solved to form a library of independent modules. This property is used to reduce inertial parameters and external forces in each structural group (module) that forms the mechanism. The reduction is propagated from module to module until the driving link is reached, and then the forward dynamics is solved analyzing exclusively that link. The presented GP method has a flexibility which is comparable with respect to the GP joint-based methods. The method is applied to the forward dynamics of a six-bar (Watt) mechanism with results that are comparable to the commercial software for Multibody Systems (MBS) dynamics.
\end{abstract}

Keywords: Forward dynamics, mechanism, modular analysis. 


\section{Introduction}

Nikravesh (1988) and Hansen (1996) classify the methods for computer aided analysis of mechanical systems into two categories: i) Special Purpose (SP), and ii) General Purpose (GP).

The GP methods are codified as libraries without specific mechanisms, though they include the elements necessary to assemble these mechanisms virtually. The modules that constitute a GP library may be developed on the concept of kinematic joint, as in the majority of commercial software for Multibody System (MBS) dynamics, or on the concept of kinematic unit. In case of planar mechanisms, the kinematic unit corresponds with a structural kinematic chain which is conventionally called Assur group. A mechanism may be designed as a sequential union of one or more driving links and one or more Assur groups.

This study presents a contribution to the dynamics of planar mechanisms. The developed method is a GP method based on kinematic units. Given that each kinematic unit is statically determinate, then it is possible to find an independent solution for its kinematics as well as for its kinetostatic analysis (inverse dynamics). Kinematical and kinetostatical independence are a basis for the modular method, which is extended in this study to the simulation (forward dynamics). The proposed method consists in a modular reduction of inertial parameters and forces, beginning with the last kinematic unit in the formation sequence of the mechanism, and propagating the reduction until the driving link (reduced link) is reached. The reduction is carried out as a function of the generalized coordinate and it is independent of time and velocity of the system. Finally, the forward dynamics is solved exclusively for the reduced link as a function of the generalized coordinate and the initial conditions.

The following references discuss GP methods for kinematics, which are based on kinematic units: Cavic et al. (2007) show an iterative method for the kinematics of high-class Assur groups (higher than a dyad), in which high-class groups are approximated by a set of dyads that should be geometrically adjusted to describe the displacement of the original group. Zhang et al.
(2006) present an alternative to the study of Cavic et al. (2007), in which the solution of the position is solved by means of an iterative algorithm by virtual search. Buśkiewicz (2006) and Calle et al. (2001) show general and optimized methods for calculating the kinematics of a mechanism, given its rule for the Assur group formation. Modular reductions of mass and force analyzed in this paper require a kinematics library; this library was adopted from the study of Calle et al. (2001). The library is a unit based GP and it is also used for the post-processing in dynamics since the calculated movement of the reduced link should be extended towards the other Assur groups. Durango (2007), Marghitu \& Crocker (2001) and Bràt \& Lederer (1973) present GP methods for inverse dynamics based on kinematic units where the friction in joints is assumed to be negligible. In general, these methods are reduced to the solution of a linear set of equations that represent dynamic equilibrium in the system. In contrast to the studies of Durango (2007), Marghitu \& Crocker (2001) and Bràt \& Lederer (1973); in the analysis made by Ruiz et al. (2010), a GP method is extended to include the effects of friction in the joints using a successive approximation method for the force analysis. None of these studies discusses the problem related to the solution of differential equations of the system's motion, i.e. a forward dynamics problem.

Wang et al. (2008) describe a modular dynamics of mechanisms using Assur groups and a state space representation which increases the computational expenses since it requires a simultaneous solution of differential equations of the motion for all the kinematic units that form the mechanism. Hansen (1996) also develops a GP method based on kinematic units in which the dynamic calculations are built on a correction of driving links' accelerations under a given state of load. Once the accelerations are calculated, then numerical integration is done in order to solve position and acceleration and to advance to the following time step. This methodology is similar to the one presented in this paper. However, there is a difference in the calculation of the driving link's acceleration: while Hansen (1996) calculates the accelerations by an iterative correction process, the method shown in this study requires to apply a procedure of force and inertial parameters 
reduction whose equations are closed and explicit, decreasing thus the computational expenses. On the other hand, the method developed by Hansen (1996) allows to calculate the dynamics of mechanisms with several Degrees of Freedom (DOF) and with a floating drive, e.g. mechanisms in which the engine is situated between two moving links; meanwhile the current study is limited to mechanisms with one DOF where the driving link has a fixed point. This study is meant to be extended in the future to mechanisms with various DOFs including floating drive.

The described reduction method conserves the advantages of GP methods based on bodies and joints and based on kinematic units: it is flexible (allows to analyze huge groups of mechanisms with only a few modules) and computationally efficient (reduced equations for each module are closed expressions). An additional advantage of this modular approach for planar mechanisms dynamics is the simplification of the solution of differential equations of motion, i.e. the analysis has been reduced to a single link which may be modelled as a body rotating around a fixed axis or as a translational body, as appropriate.

\section{Methodology for modular dynamics}

Dynamical simulation or time response analysis is a problem in which a mechanism with known geometry and inertial properties is loaded by a set of external and driving forces (e.g. functions of generalized coordinate or couple - velocity functions). The result of this analysis is the mechanism kinematics, i.e. positions, velocities and accelerations of the links as functions of time. The simulation requires numerical integration of differential equations describing the motion of the system; and this defines its complexity level.

This article presents a GP method based on kinematic units for the forward dynamics of mechanisms with one DOF. Kinematic units are independent and reusable modules for analysis, representing a mechanism when they are assembled in an appropriate sequence. A collection of kinematic units forms a library, which, in this work, was implemented following the structural group concept developed by Assur (1913), i.e. kinematic units whose characteristic is that they do not have any effect on the DOF of the mechanism. The Assur groups constitute analysis units which are independent from the point of view of the kinematics as well as the kinetostatics. Here, this property is extended to the force and inertial parameters reduction: each group can be reduced independently, and the reduction may be propagated up to the driving link. The described reduction of forces is based on the power equivalence, while the inertial parameters reduction establishes kinetic

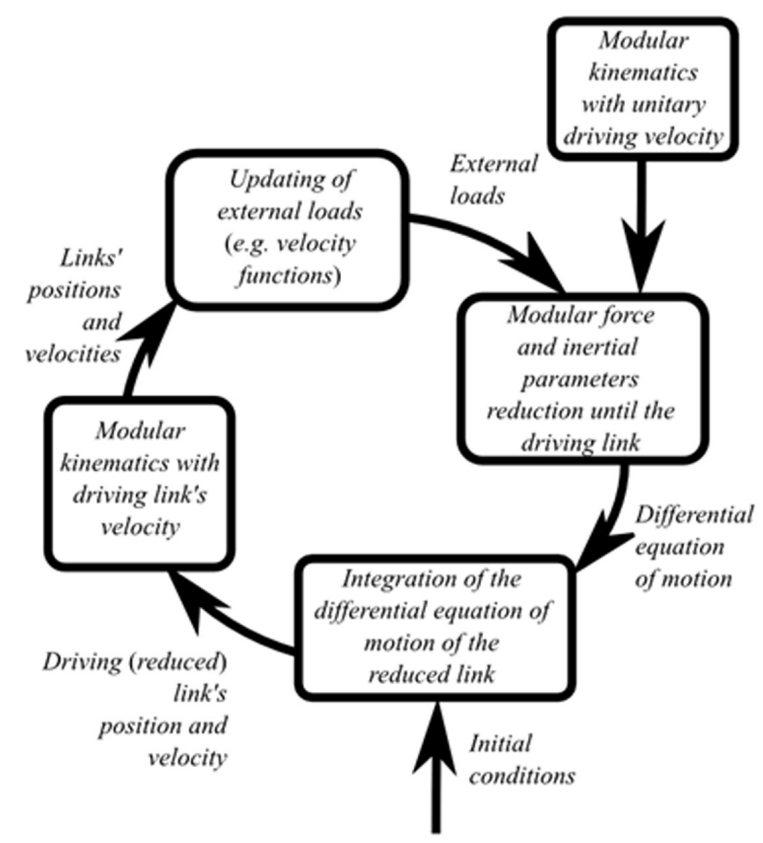

Figure 1. Modular dynamics of mechanisms. 
energy equivalence between the system and its reduced equivalent. The method for the analysis is shown in Figure 1 and it is also explained in the following points:

i) Modular kinematics with unitary driving velocity. Velocities of all the links and points of interest (centers of mass, force application points) are calculated using the modular method described by Calle et al. (2001). The analysis starts with the driving link, which is assumed to have a unitary velocity. The solution, then proceeds following the sequence of mechanism formation towards the other modules as it is shown in Figure 2. Given that each module is kinematically determined, therefore it has one independent solution.

ii) Modular reduction of forces and inertial parameters up to the driving link. Given the kinematics calculated in (i), and knowing the loads and inertial parameters of each group, the reduction of every kinematic unit is calculated the way towards the link which connects this unit with the kinematic unit directly preceding in the formation sequence, see Figure 2. This sequence is adopted to establish a convention that would facilitate computational implementation. However, this reduction order is not strict since the groups may be reduced without any particular order and directly to the reduced link. The process of reduction simplifies the system into the driving link through the power and kinetic energy equivalence. Thus, energy and power are related by means of the virtual work principle. Eq. (1) is obtained for a reduced link in rotation:

$$
d\left(\frac{J_{r e d}(q) \omega(q)^{2}}{2}\right)=\left(\tau(q)+\tau_{r e d}(q)\right) d q
$$

where $q$ is the generalized coordinate, $\omega$ is the angular velocity, $\tau$ is the driving torque, $\tau_{\text {red }}$ is the reduced couple, and $J_{\text {red }}$ is the reduced inertial moment. For obtaining the angular acceleration $\zeta$ Eq. (1) is differentiated and $J_{\text {red }}$ is approximated by finite differences between $q_{i}$ and $q_{i}+1$, then Eq. (2) is obtained:

$$
\xi\left(q_{i}\right) \approx \frac{\tau\left(q_{i}\right)+\tau_{\text {red }}\left(q_{i}\right)-\frac{1}{2} \frac{\omega\left(q_{i}\right)^{2}\left(J_{r e d}\left(q_{i+1}\right)-J_{r e d}\left(q_{i}\right)\right)}{\Delta}}{J_{r e d}\left(q_{i}\right)}
$$

where $\Delta$ is the discretization step in radians. Linear acceleration of a translational reduced link is obtained analogously.

iii) Integration of the differential equation of motion. Given the initial conditions of

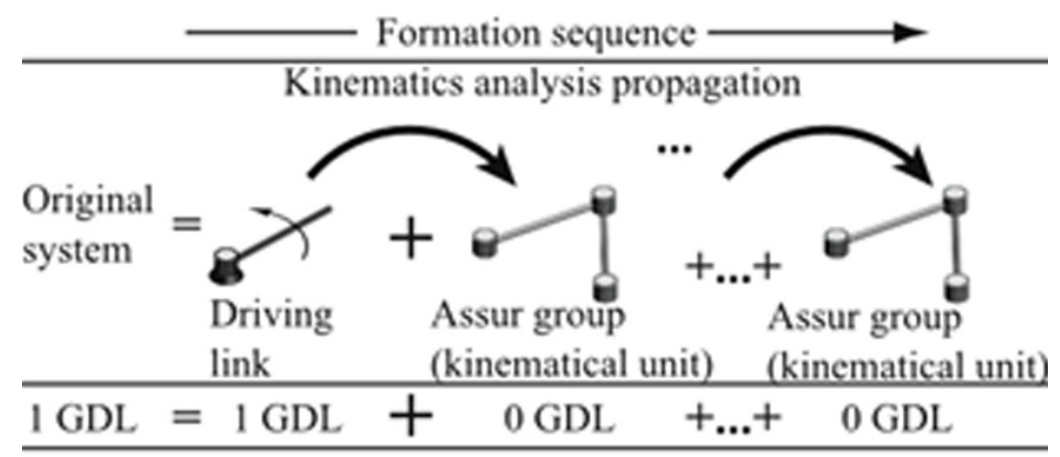

Reduced force and reduced inertial parameters propagation

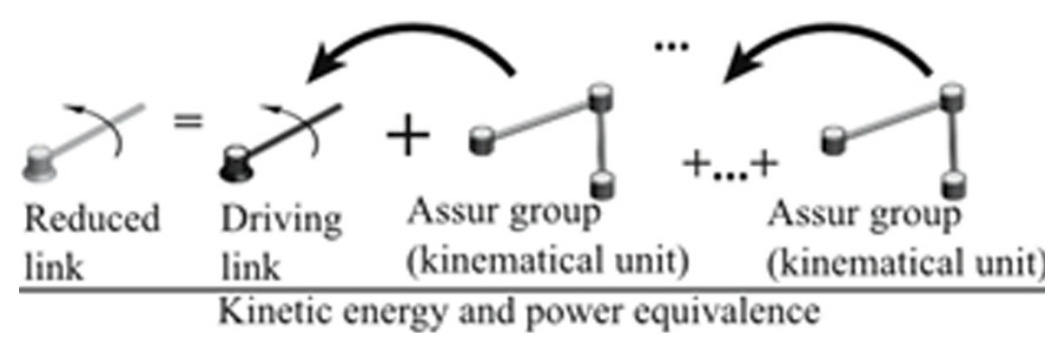

Figure 2. Modular propagation of the kinematic analysis and of the reduction of forces and inertial parameters in mechanisms. 
motion, the differential equation obtained in (ii) is numerically integrated with respect to the generalized coordinate. The result of the integration will be the driving link's velocity, which is then applied in the following step of generalized coordinate discretization. This point is one of the advantages of this method as the mechanism has been reduced to one single differential equation instead of a system of differential equations.

iv) Modular kinematics with reduced link velocity. The velocity calculated in (iii) is propagated until it reaches the last kinematic unit, as it is shown in Figure 2. Velocities of all the links are obtained.

v) Updating of external forces. Given the velocities calculated in (iv) and knowing the generalized coordinate, the values of external forces dependent on the velocity, generalized coordinate, or time, are updated. Now, it is possible to return to the step (ii) and continue with the next integration.

The following section develops the modular dynamics methodology for the case study of mechanisms with driving link in rotation and one or more Assur groups of two links with rotational pairs. Other results may be obtained analogously for mechanisms with driving link in translation or mechanisms formed by other Assur groups.

\section{Results and discussion}

\subsection{Force reduction}

For the force reduction, there are given external forces and external couples which act in each kinematic unit. The principle of virtual displacements is used in this reduction; by this principle, power equivalence is obtained between the original system and its reduced equivalent.

Assur group with two links and rotational pairs. Figure 3.a presents an Assur group with two links and rotational pairs where link 3 is assumed to be the reduced link. It is also assumed that the driving link has a fixed pivot and therefore the system of forces and couples is diminished to a single reduced couple, which acts instead of the

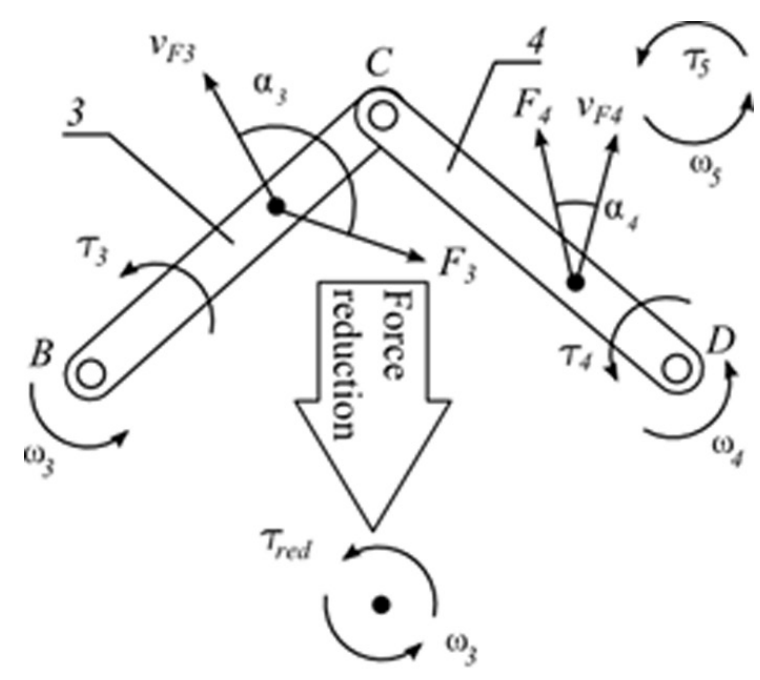

(a)

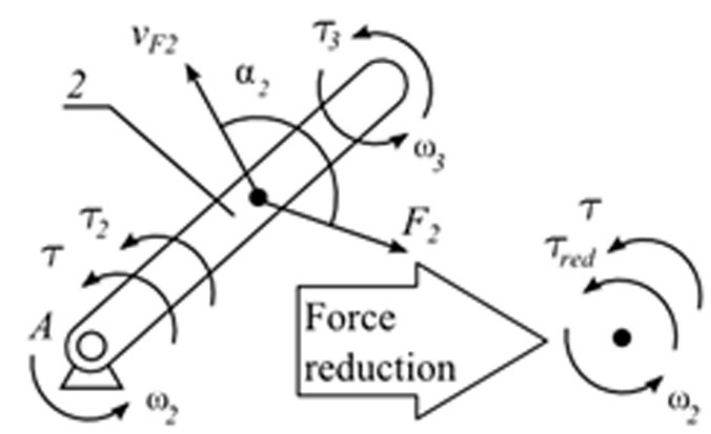

(b)

Figure 3. Force reduction. a) In an Assur group with two links and rotational pairs. b) In a driving link with fixed pivot $A$.

set of external loads. Eq. (3) represents power equality for the calculation of the reduced couple:

$$
\begin{gathered}
\tau_{\text {red }}=F_{3} \frac{v_{F 3}}{\omega_{3}} \cos \alpha_{3}+F_{4} \frac{v_{F 4}}{\omega_{3}} \cos \alpha_{4}+ \\
\tau_{3}+\tau_{4} \frac{\omega_{4}}{\omega_{3}}+\tau_{5} \frac{\omega_{5}}{\omega_{3}}
\end{gathered}
$$

where $F_{j}$ represents the magnitude of the load on the link $j, \tau_{j}$ is the couple on the link $j, v_{F j}$ is the velocity magnitude of the application point of the $j$-th load, $\alpha_{j}$ is the angle between the force and the velocity of its application point, $j=3$, 4 for Figure 3.a and $\tau_{5}$ represents the reduced couple that comes from the group preceding in the reduction propagation.

Driving link with fixed pivot. Figure 3.b presents a driving link with fixed pivot. This is the 
reduced link of the whole mechanism. Eq. (4) represents the power equivalence of the system for calculation of the reduced couple, which acts on the driving link:

$$
\tau_{\text {red }}=F_{2} \frac{v_{F 2}}{\omega_{2}} \cos \alpha_{2}+\tau_{2}+\tau_{3} \frac{\omega_{3}}{\omega_{2}}
$$

where $F_{2}$ is the magnitude of external force on the link, $\tau_{2}$ represents the action of external couples that act on the link without including the driving torque, $v_{F 2}$ is the velocity of the application point of $F_{2}, \alpha_{2}$ is the angle between external force and velocity of the application point, and $\tau_{3}$ represents the reduced couple that comes from the group preceding in the reduction.

\subsection{Inertial parameters reduction}

The reduction of inertial parameters is calculated by means of equivalence in energy between the original system and the reduced one. The reduction is a function of link velocity ratios; therefore it is determined from a modular kinematics with unitary driving velocity. Inertial parameters of all the links are given.

Assur group with two links and rotational pairs. Figure 4.a shows an Assur group with two links and rotational pairs where the link 3 is assumed to be the reduced link. It is also supposed that the driving link has a fixed pivot and therefore the set of inertial parameters is diminished to a single reduced moment of inertia $J_{\text {red }}$ which represents the dyad. Eq. (5) describes the kinetic energy equivalence for calculation of the reduced moment of inertia:

$$
\begin{gathered}
J_{\text {red }}=m_{3}\left(\frac{v_{G 3}}{\omega_{3}}\right)^{2}+m_{4}\left(\frac{v_{G 4}}{\omega_{3}}\right)^{2} \\
+J_{3}+J_{4}\left(\frac{\omega_{4}}{\omega_{3}}\right)^{2}+J_{5}\left(\frac{\omega_{5}}{\omega_{3}}\right)^{2}
\end{gathered}
$$

where $m_{j}$ is the mass of the link $j, v_{G j}$ is the velocity of the center of mass $j, j=3,4$ for Figure 4. a and $J_{5}$ is the reduced moment of inertia that comes from the group preceding in the reduction propagation.

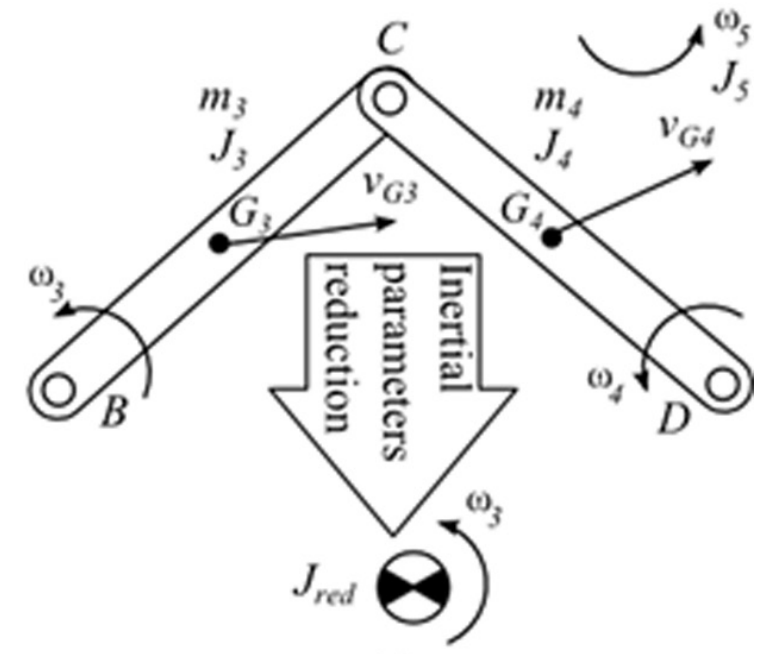

(a)

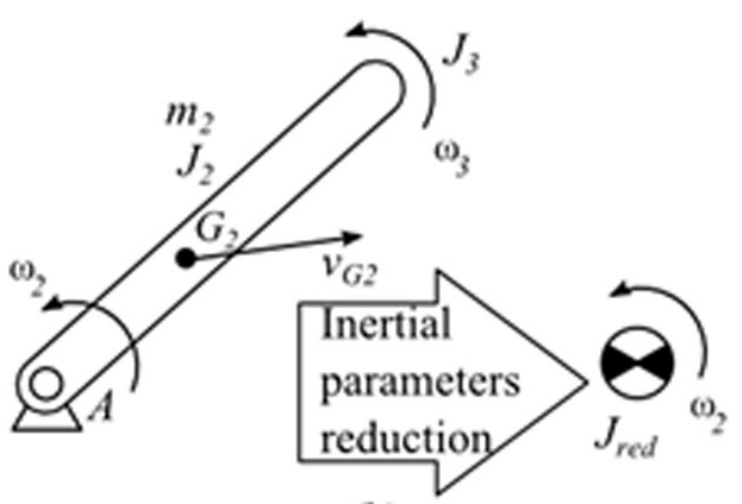

(b)

Figure 4. Inertial parameters reduction. a) In an Assur group with two links and three rotational pairs. b) In a driving link with fixed pivot $A$.

Driving link with fixed pivot. Figure 4.b shows a driving link with fixed pivot. This link has been assumed to be the reduced link of the whole mechanism. Eq. (6) represents the equivalence of the system's kinetic energy for calculation of the reduced moment of inertia:

$$
J_{\text {red }}=m_{2}\left(\frac{v_{G 2}}{\omega_{2}}\right)^{2}+J_{2}+J_{3}\left(\frac{\omega_{3}}{\omega_{2}}\right)^{2}
$$

where $m_{j}$ and $J_{2}$ stand for inertial parameters of the link, $v_{G 2}$ is the center of mass velocity and $\mathrm{J} 3$ represents the reduced moment of inertia that comes from the group preceding in the reduction propagation.

The reduction of forces and inertial parameters for an Assur group with two links and three 
Table 1. Geometrical and inertial parameters of the case study.

\begin{tabular}{cccccc}
\hline Parameter & $\begin{array}{c}\text { Value } \\
{[\mathbf{m}]}\end{array}$ & Parameter & $\begin{array}{c}\text { Value } \\
{[\mathbf{k g}]}\end{array}$ & Parameter & $\begin{array}{c}\text { Value } \\
{\left[\mathbf{k g} \mathbf{m}^{2}\right]}\end{array}$ \\
\hline$l_{O 2 A}$ & 1,0 & $m_{2}$ & 1,0 & $J_{2}$ & 0,0833 \\
$l_{A B}$ & 1,5 & $m_{3}$ & 1,5 & $J_{3}$ & 0,2813 \\
$l_{B O 4}$ & 1,5 & $m_{4}$ & 1,5 & $J_{4}$ & 0,2813 \\
$l_{C E}$ & 1,0 & $m_{5}$ & 1,0 & $J_{5}$ & 0,0833 \\
$l_{D E}$ & 1,0 & $m_{6}$ & 1,0 & $J_{6}$ & 0,0833 \\
$l_{O 2 O 4}$ & 1,6 & & & & \\
\hline
\end{tabular}

rotational pairs and for a driving link with fixed pivot is described in the sections 3.1 and 3.2. These reductions constitute two modules in the library of modular dynamics of planar mechanisms. The modules may be assembled and recycled in the simulation of diverse mechanisms. It is necessary to develop additional modules for the formation sequences that require other structural groups. The flexibility is lower than in case of GP programs based on joints in which the combination of a few modules allows simulation of wide groups of mechanisms. However, just a few modules based on kinematic units are enough to study the majority of mechanisms used in modern technic, thereby minimizing the difference with respect to programs based on joints, and proving the flexibility reported by Hansen (1996).

In terms of computational efficiency, the reduced equations are characterized by being algebraic and explicit, e.g. Eq. (3) and Eq. (5) for an Assur group with two links and rotational pairs. In case of mechanisms with one DOF the system is represented, by reduction, by means of one single differential equation. This is an advantage when compared with strategies that require the solution of a system of differential equations, as

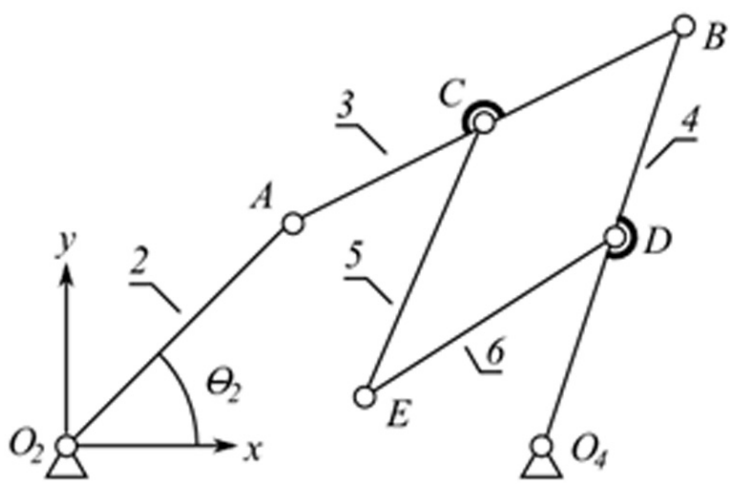

Figure 5. Case study, Watt's mechanism. in the study of Wang et al. (2008), or that require iterations to correct the driving acceleration in addition to solving the differential equation, as for example Hansen (1996). The following section presents forward dynamics of a six-bar mechanism using the modular approach.

\subsection{Case study}

Figure 5 shows a Watt's planar six-bar mechanism with geometric and inertial parameters registered in Table 1. The mechanism starts its motion from rest under the action of its weight at and with negligible friction. The simulation is being developed until the mechanism reaches the standstill again.

The formation sequence of the mechanism is: An Assur group formed by links 3 and 4 is added to the driving link 2 between the points A and O4, and finally a second Assur group formed by links 5 and 6 is added between the points $\mathrm{C}$ and $\mathrm{D}$, as it is shown in the graph (structural diagram) (7):

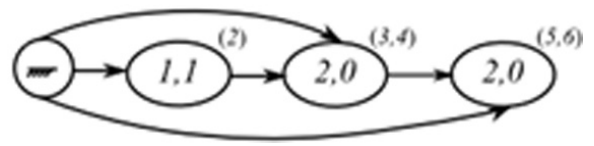

where vertices represents the structural groups (fixed and driving link and Assur groups) and edges represents the group connections, e.g. vertex $2,0^{(5,6)}$ represents a module formed by 2 links (links 5 and 6) and which adds 0 DOF to the kinematical structure, this is, the module is an Assur group. Both Assur groups, vertices $2,0^{(3,4)}$ and 2, $0^{(5,6)}$, have the same topology and are represented by the same module. The dynamic simulation is carried out following the methodology described in section 2 and in 
Figure 1. The required modules correspond to the kinematics and to the reduction of forces and inertial parameters for a driving link with fixed pivot, and for an Assur group with two links and three rotational pairs.

The integration of the differential equation of motion is calculated assuming that the acceleration is constant for small steps (Euler's integration). The size of the adopted step was $1 \times 10^{-3} \mathrm{rad}$, which is, in fact, a small size. Since the focus of this study is the modular approach, no other numerical methods were considered. To diminish the discretization and improve the efficiency, multistep methods may be implemented, as for example the Runge-Kutta methods; this is proposed for a future study. The results are registered in Figure 6, describing the forward dynamics of the driving link in terms

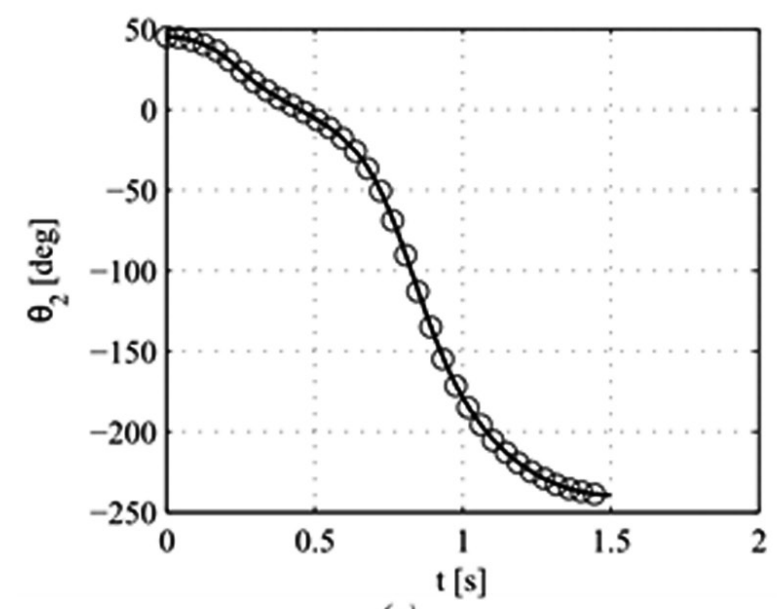

(a) of its angular displacement (Figure 6.a) and its angular velocity (Figure 6.b). The results are also presented applying commercial MBS software based on joints. Additionally, Figure 6.c shows the magnitude of the reactions in the pairs $\mathrm{O}_{2}$, $\mathrm{A}$, and $\mathrm{E}$, which were calculated by the modular method. The sequence of the force calculation is contrary to the sequence of formation of the mechanism described in (7).

Figure 7 presents a validation of the simulation by means of energy conservation. The conservation is formulated in the absence of all external forces different from the weight $\Delta \mu_{g}$, it is registered in Eq. (8):

where $\Delta T$ is the change in kinetic energy and is the change in potential energy of the system,

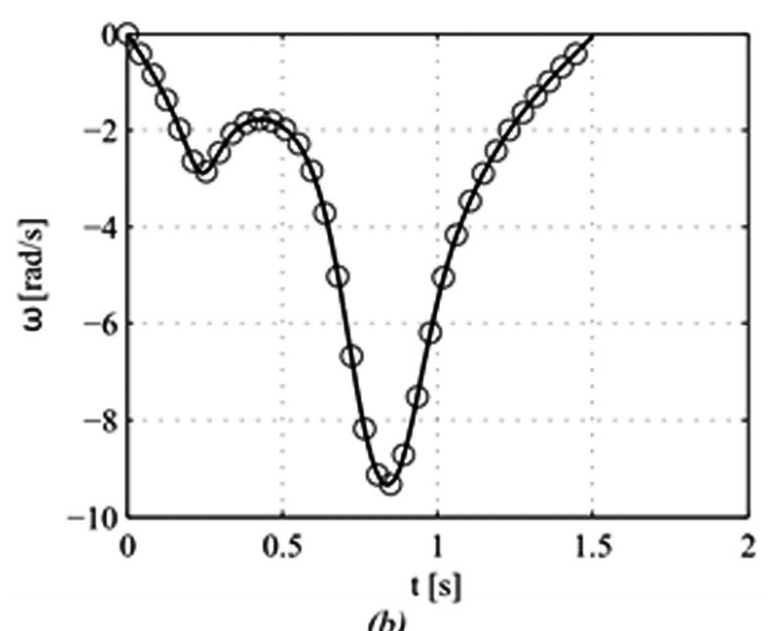

(b)

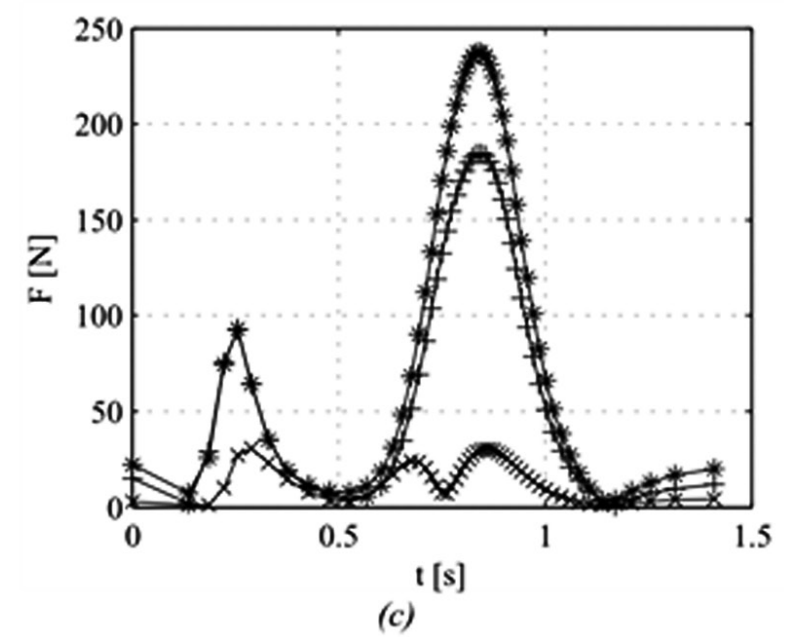

Figure 6. Forward dynamics of the case study's reduced link. a) Generalized coordinate ( $\theta$ 2). b) Angular velocity ( $\omega)$. c) Pair reactions $(F)$. Convention for the figures a) and $b)$ : - modular dynamics; $\mathbf{O}$ commercial MBS. Convention for the figure c): * reaction in $O 2 ;+$ reaction in $A$; $x$ reaction in $E$. 


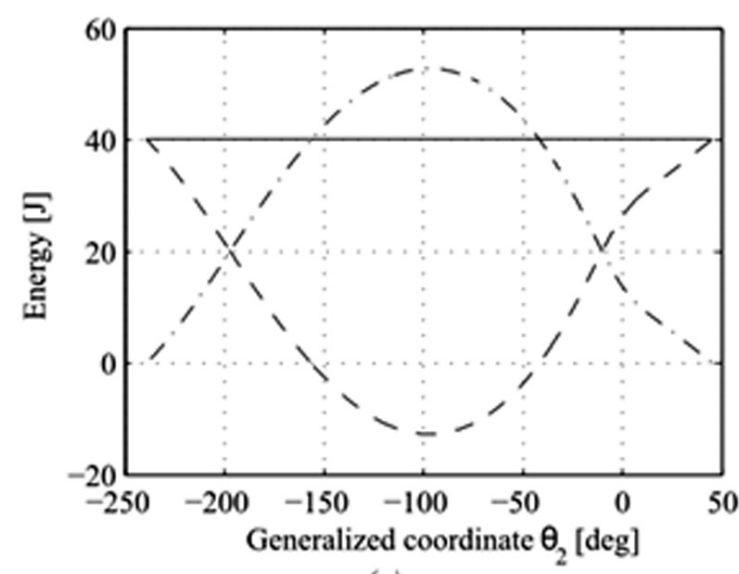

(a)

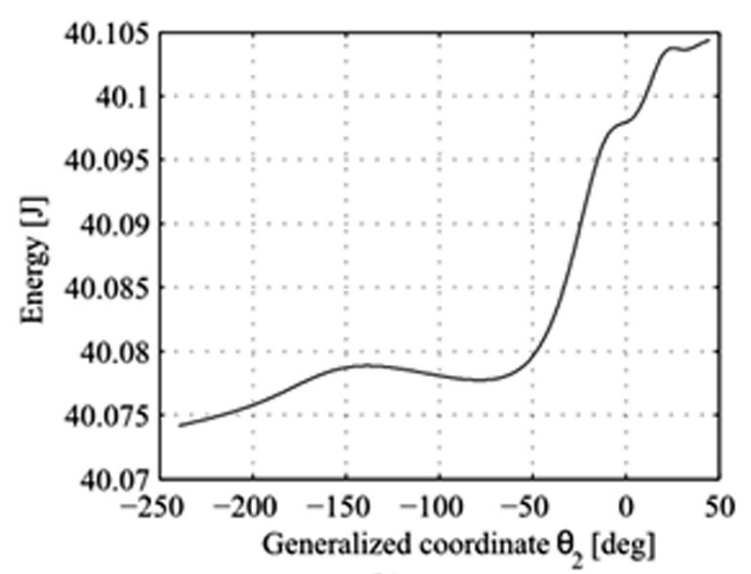

(b)

Figure 7. Energy variation for the case study. a) Kinetic and potential energy. b) Total energy. Convention: $-\quad T$, kinetic energy;_._._V , potential energy; _ $E=T+V$.

taking as reference the $O x y$ system from Figure 5. Figure 7.b presents the details on the energy variation which is explained partially by the application of Euler's integration.

\section{Conclusions}

In this study, there has been presented a contribution to the modular dynamics of planar mechanisms with one DOF. The methodology, described in Figure 1, is based on the concept of kinematic unit, which allows formulation of independent solutions (modules) for the kinematics and for the reduction of forces and inertial parameters. A minimal expression of the system, in the form of an ordinary differential equation, is obtained systematically by means of modular reduction of forces and inertial parameters. The behavior of the mechanism is therefore represented by the driving link under the action of the driving force and a reduced force and a reduced inertia, which altogether directly describe the system's dynamics.

This contribution keeps the flexibility of GP methods, since it is possible to simulate wide families of mechanisms by combination of few modules. This includes the majority of the mechanisms used in modern technic. It is also emphasized that the kinematic solution for each unit is tailored, which corresponds with the advantage of SP methods.

The methodology was implemented into the analysis of Watt's six-bar mechanism (section 3.3), and the results were compared with a commercial MBS software.

\section{Acknowledgements}

The authors want to thank to Ing. Juan Sebastián Aristizábal for his contribution to the early phase of this project.

\section{References}

Assur, L.V. (1913). Investigation of plane hinged mechanisms with lower pairs from the point of view of their structure and classification (in Russian): Part I, II. Bulletin of Petrograd Polytechnical Institute, 20, 329-386.

Bràt, V., \& Lederer, P. (1973). KIDYAN: Computer-aided kinematic and dynamic analysis of planar mechanisms. Mechanism and Machine Theory, 8 (4), 457-467.

Buśkiewicz, J. (2006). A method of optimization of solving a kinematic problem with the use of structural analysis algorithm (SAM). Mechanism and Machine Theory, 41 (7), 823837.

Calle, G., Quintero, H.F., \& Díaz, A. (2001). Análisis cinemático de mecanismos planos a partir del análisis estructural según Assur. In Congreso Iberoamericano de Ingeniería Mecánica CIBIM V, Mérida, Venezuela, p. 1231-1240.

Cavic, M., Kostic, M., \& Zlokolica, M. (2007). Position analysis of the high class kinematic group 
mechanism. In 12th IFToMM World Congress, Besançon, France, p. A744.

Durango, S. (2007). Dynamics of second-class planar mechanisms. In ISPE 23rd International Conference on CAD/CAM Robotics and Factories of the Future, Santafé de Bogotá, Colombia, p. 421-428.

Hansen, M.R. (1996). A general method for analysis of planar mechanisms using a modular approach. Mechanism and Machine Theory, 31 (8), 1155-1166.

Marghitu, D.B., \& Crocker, M.J. (2001). Analytical elements of mechanisms. Cambridge: Cambridge University Press.

Nikravesh, P.E. (1988). Computer aided analysis of mechanical systems. New Jersey: Prentice Hall, Inc.
Ruiz, O., Durango, S., \& Calle, G. (2010). Analytical method for the kinetostatic analysis of the second-class RRR AssurGroup allowing for friction in the kinematic pairs. Journal of the Brazilian Society of Mechanical Sciences and Engineering, 32 (3), 200-207.

Wang, H., Lin, Z., \& Lai, X. (2008). Composite modeling method in dynamics of planar mechanical system. Science in China-Series E: Technological Sciences, 51 (45), 576-590.

Zhang, Q., Zou, H.J., \& Guo, W.Z. (2006). Position analysis of higher-class Assur groups by virtual variable searching and its application in a multifunction domestic sewing machine. The International Journal of Advanced Manufacturing Technology, 28 (5-6), 602-609. 